\title{
Recent Advances in Dental Implantology
}

\author{
Dr. G. Ajay kumar \\ Reader, Army College of dental sciences, secunderabad, Andhra pradesh.
}

\begin{abstract}
Dental implant treatment is gaining increasing acceptance by Patients. There is wide acceptance in the adopting a minimal invasive approach on restorative dentistry and when combined with the improvement in reliability of implants, dental implant treatment has been transformed from the last choice to the first choice of treatment being offered to patients.

With the increasing research both in basic science and clinical trials coupled with improvement in dental implant designs, the success rate of the dental implants has been steadily improving to over $95 \%$. The success rate is better in denser bone and particularly in the anterior mandible; the success rate reaches $99 \%$. The critical factors of success in the dental implant treatment are related to the training of surgeons, the design of implants, the intra-operative control of implant placement, the consideration of the occlusal loading and the long term maintenance. The present paper reviews the recent advances in the field of implantology.

Key Words: Implant, clinical trials, design, occlusal loading
\end{abstract}

\section{Introduction}

In the dental implant placement, imaging is the key to successful case planning ${ }^{1}$. The Latest technology in dental imaging is Cone Beam Computed Tomography CBCT which implements revolutionary 3D Images with axial, coronal, and sagittal views and a stream of useful data that the doctor can reconstruct as in the form of images when needed, while delivering substantially less amounts of radiation to the patient.

Recent Advances in Computed Tomography are ${ }^{2}$

Cone beam ct: It uses a conical beam and reconstructs the image in any direction using special software. It gives all the information of a CT but, at $1 / 8^{\text {th }}$ the radiation dose and at a lower cost.

Microtomograph: another modification of CT is especially useful in acquiring serial sections of bone-implant interface.

Multislice helical ct: It offers higher accuracy of images when compared to CT, the advantages of this type of imaging were evident and the limitations of delivery clear, which spawned the development of a number of techniques, referred to generically as 'Dentascan Imaging'.

Interactive computed tomography: This technique enables the radiologist to transfer the imaging study to the clinician as a computer file and enables the clinician to view and interact on their own computer. An important feature of ICT is that the clinician and radiologist can perform "electronic surgery" (ES) by selecting and placing arbitrary size cylinders that simulate root form implants in the images.

Magnetic resonance imaging: MR imaging is a three-dimensional imaging technique with an electronic image acquisition process and a resulting digital image.

Changes in implant shapes, sizes, materials, and coatings along with technique innovations, such as guided tissue regeneration and immediate loading concepts, have expanded the indications for dental implant therapy.

The design of implants has improved a lot by research on the surface treatment of dental implants that increases the ratio of bone to implant contact such as plasma sprayed surface ${ }^{3}$, Ion-sputtering coating, Anodized surface, Sandblasted and acid etched, Hydroxyapatite coating and biochemical surface modification is to immobilize proteins, enzymes/ peptides on biomaterial for the purpose of inducing specific cells and tissue response or in other words to control the tissue implant interface with molecules delivered directly to the interface. $^{4}$

The configuration of implants in achieving better primary stability is so critical to the osseointegration process $^{5}$; the range of prosthetic abutments that can handle any implant malalignment, The intra-operative control of implant placement is much better by the refinement of drilling machines in controlling the drill speed and torque control in reducing the overheating of the bone surrounding the implants; the control of water irrigation or the availability of internal implant irrigation systems in further minimising the rise in bone 
temperature; the sharpness of drills by having more disposable drills being available ${ }^{6}$; custom-made surgical splint in defining the implant location and angulation based on CT data rather than plain radiography.

The dentists and prosthodontists are more conscious of the occlusal loading on the dental implant prosthesis ${ }^{7}$. Most patients demand for dental implant fixed bridges and that require good precision in dental implant alignment and the implants to be linked up in triangular configuration in enhancing the stability of the implant support bridges to resist from the lateral displacement forces. The choice of acrylic, porcelain or newer materials depend on both the occlusal loading and aesthetic requirements.

\section{Peri implant surgery}

The loss of teeth will result in a concomitant resorption of the alveolar bone and with time, there may be insufficient bone height or width for the placement of implants. and this is being known that adequate implant length and width are important to improve the longevity of dental implants. Various bone augmentation methods ${ }^{8}$ from simple onlay bone graft for small depression, inlay bone graft for sandwich osteotomy to the maxillary sinus floor augmentation are developed for enhancing the bone volume. One of the newest procedures for augmenting areas of bone is called distraction osteogenesis. Osteoinductive and osteoconductive substances are now available to assist in accelerating healing and present great promise for future applications.

When there is a lack of soft tissue due to atrophy or from ablative surgery, vestibuloplasty and palatal graft transplant are gaining wider acceptance. The use of free gingival graft transplant has been developed and was found to be technically easier and produced less morbidity to the patients. Different peri-implant surgeries may be combined to treat the problems related to either the bone or soft tissue deficiency around the implant placement and in implant maintenance.

\section{Development in image guided implantology}

Image guided implant placement ${ }^{9}$ has undergone revolutionary development in the last few years. There are essentially two main types of image guided implant surgery. Both types involve the implant planning on dedicated softwares to define the position and angulation of the implants to be placed, the avoidance of contacting the inferior alveolar nerve or the intrusion into the maxillary sinus. The difference is one is a real time navigational implant surgery whereas the second one is the insertion of implants with the use of a stereolithographic surgical splint.

Both systems have benefits and drawbacks. The benefits of the real time navigation is that the implant placement is being guided via computer onscreen guidance and intra-operative adjustment can be made if the implant position in bone is found to deviate from the computer planned position. The drawbacks are the expensive machine and long calibration time that makes the productivity not very economical. The system is good for teaching as training tool rather than being used in busy clinics. In contrast, the computer designed surgical splints enhance the speed of implant placement tremendously. The drawback is that if there is any error in the computerised planning or splint fabrication, the surgeons cannot make any intra-operative adjustment unless the surgeons abandon the use of the splint at all and the implants will ultimately be wrongly placed.

In addition to the placement of the dental implants on the alveolar bone, very long implants of 40$55 \mathrm{~mm}$ are available to insert through the palatal bone to the zygoma. These zygomatic implants are extremely reliable and with the advantage of avoiding any sinus grafting and bone graft.Implants can be placed extra-orally for supported facial or ear prostheses. With the navigational surgery, this will enhance the accuracy of implant placement. Research is being undertaken to apply CAD-CAM method for facial prosthesis construction

\section{Future development of dental implantology}

Improvements such as the coating of the implants with bone growth factors are beingactively researched in an attempt to increase the speed of implant osseointegration and to enhance the longevity of the implants. The Growth Factors are natural proteins found in our bodies that stimulate growth of certain tissues. With respect to bone, genetic engineers have been able to isolate and clone Bone Morphogenic Proteins (BMPs), which have been shown to induce tremendous bone growth in many animal and recently human clinical studies. BMPs may very well become a potential substitute for autogenous graft material for certain applications in the future.

Research in substituting the titanium material with ceramic is being pursued in order to reduce the exposure of the metallic implant body above the gingival level. This is to a certain extent being improved by the recently developed zirconium material, which is opaquely white and extremely hard and is a suitable material for the abutment construction. A material of better translucency is recently available and is composed of alumina but is considered too brittle for posterior bridge construction. There is little doubt for further search of a biological compatible material simulating the tooth colour for dental implants. We hope that this will not be too long before the opportunity of tissue engineered teeth by cell culture methods. 
Long term maintenance of dental implants is gaining importance as the main factor affecting the long term prognosis of dental implants. Patients should be well instructed in maintaining oral hygiene around the dental implants. A lot of implant cleansing aids are more widely available in line with the growing demand of dental implants. The presence of attached gingiva is being recognised as important for the maintenance of good oral hygiene in reducing the incidence of periodontal disease around the implants (peri-implantitis). The patients are better informed in committing to regular oral hygiene visits every 6 months and annual check-up with either the surgeons or the prosthodontists.

\section{References}

[1] Frederiksen NL. Diagnostic imaging in dental implantology. Oral Surgery Oral Medicine Oral Pathology 1995;80:540-54.

[2] Potter BJ, Shrout MK. Implant site assessment using cross-sectional tomographic image. Oral Surgery Oral Medicine Oral Pathology

[3] 1997;84:436-41.

[4] Baier RE, Meyer AE. Implant surface preparation. Int J Oral Maxillofac Implants 3:9-20,1998.

[5] David A. Puleo, Mark V. Thomas: Implant Surfaces. Dent Clin N Am 50:323-338, 2006

[6] Sennerby L, Roos J. Surgical determinants of clinical success of osseointegrated oral implants: a review of the literature. Int J Prosthodont

[7] 1998; 11(5): 408-20

[8] Glauser R, Portmann M, Ruhstaller P, Gottlow J, Schärer P. Initial implant stability using different implant designs and surgical techniques.

[9] A comparative clinical study using insertion torque and resonance frequency analysis. Appl Osseointegration Res 2001a;2(1): 6-8.

[10] Gross MD. Occlusion in implant dentistry. A review of the literature of prosthetic determinants and current concepts. Aust Dent J. 2008 Jun;53

[11] Suppl 1:S60-8

[12] Aichelmann-Reidy ME, Yukna RA. Bone replacement grafts. Dent Clin North Am 1998;42:491-503.

[13] Kopp, K.C., Koslow, A.H. \& Abdo, O.S Predictable implant placement with a diagnostic/ surgical template and advanced radiographic

[14] imaging. Journal of Prosthetic Dentistry2003, 89: 611-615.

[15] Kengo Shimono, Masamitsu Oshima et al. The effect of growth factors for bone augmentation to enable dental implant placement: A

[16] systematic review Japanese Dental Science Review, Volume 46, Issue 1, February 2010, Pages 43-53 